Descriptive statistics (frequencies, means and standard deviations) were calculated using SPSS version 12.

Results A total of 141 patients (table 1), who fulfilled the SLICC 2012 Classification Criteria for SLE were enrolled in the study. The mean age of the population was 39.8 with a standard deviation of 13.8. 136 patients (96.5\%) were female. Analysis of the immunologic abnormalities revealed a positive ANA test in 139 (99.3\%), and a positive anti-dsDNA test in $80(57.6 \%)$ of the patients, for whom results were available. Synovitis involving two or more joints was reported by 120 $(85.1 \%)$ of the patients, followed by acute cutaneous lupus 57 $(40.4 \%)$ and non-scarring alopecia 55 (39.0\%). Of the 32 patients with renal involvement, 28 patients had a result for an anti-dsDNA. test, of which 22 (78.6\%) were positive.

Conclusion The female to male ratio was comparable to other Asian populations. Our study population had similar clinical and immunologic findings as compared to published literature. A significant proportion of patients who had renal involvement had a positive Anti-dsDNA test. Future longitudinal studies would allow causal inferences to be made.

Acknowledgement The Authors will like to thank all doctors and clerical staff who worked in the Rheumatology Unit during the study period, for their assistance with data collection. Special thanks to Mr Darien Wong and Mr Jared Ramkissoon who assisted with the database. Thanks to Dr Peter Poon King and all consultants at the SWRHA.

\section{CS-18 CEREBROVASCULAR EVENTS IN SYSTEMIC LUPUS ERYTHEMATOSUS: RESULTS FROM AN INTERNATIONAL, INCEPTION COHORT STUDY}

John G Hanly*. Dalhousie University and Queen Elizabeth II Health Sciences Center, Halifax, Nova Scotia, Canada

\subsection{6/lupus-2018-Ism.53}

Background Vascular disease, including involvement of the cerebral circulation, is a frequent cause of morbidity and mortality in SLE. Cerebrovascular events (CerVEs) are reported in $5-18 \%$ of patients in previous cohort studies. Potential etiologies include procoagulant factors due to SLE (e.g. antiphospholipid antibodies, endothelial activation and vasculitis) and factors which promote accelerated atherosclerosis (e.g. hypertension, hyperlipidemia and SLE itself). The relative contribution of these factors and the outcome of clinical CerVEs in a general lupus population have not been well documented.

Objective To determine the frequency, associations and outcomes of cerebrovascular events (CerVEs) in a multi-ethnic/ racial, prospective, SLE disease inception cohort.

Methods Patients were assessed annually for 19 neuropsychiatric (NP) events including 5 types of CerVEs: (i) Stroke; (ii) Transient ischemia; (iii) Chronic multifocal ischemia; (iv) Subarachnoid/intracranial hemorrhage; (v) Sinus thrombosis. Global disease activity (SLEDAI-2K), SLICC/ACR damage index (SDI) and SF-36 scores were collected. Time to event, linear and logistic regressions and multi-state models were used as appropriate.

Results Of 1,826 SLE patients, $88.8 \%$ were female, $48.8 \%$ Caucasian, mean \pm SD age $35.1 \pm 13.3$ years, disease duration $5.6 \pm 4.2$ months and follow-up $6.6 \pm 4.1$ years. CerVEs were the fourth most frequent NP event: 82/1,826 (4.5\%) patients had 109 events, 103/109 (94.5\%) were attributed to SLE and 44/109 (40.4\%) were identified at enrollment. The predominant events were stroke [60/109 (55.0\%)] and transient ischemia [28/109 (25.7\%)]. CerVEs were associated with other NP events attributed to SLE (HR (95\% CI): (3.16; $1.73-5.75) \quad(\mathrm{p}<0.001)$, non-SLE NP $(2.60 ; 1.49-4.51)$ $(\mathrm{p}<0.001)$, African ancestry at US SLICC sites $(2.04 ; 1.01-$ 4.13) $(p=0.047)$ and organ damage $(p=0.041)$. Lupus anticoagulant increased the risk of first stroke and sinus thrombosis [2.23 (1.11, 4.45) $\mathrm{p}=0.024]$ and TIA [3.01 (1.15, 7.90) $\mathrm{p}=0.025]$. Physician assessment indicated resolution or improvement in the majority but patients reported sustained reduction in SF-36 summary and subscale scores following CerVEs $(\mathrm{P}<0.0001)$.

Conclusion CerVEs, the fourth most frequent NP event in SLE, are usually attributable to lupus. In contrast to good physician reported outcomes, patients report a sustained reduction in health-related quality of life following CerVEs.

Acknowledgements This work is presented on behalf of the Systemic Lupus International Collaborating Clinics (SLICC) and was funded in part by a grant from the Canadian Institutes of Health Research (MOP-88526) to Dr. Hanly.

\section{CS-19 HEART FAILURE HOSPITALIZATIONS AMONG SLE AND DIABETES MELLITUS PATIENTS COMPARED TO THE GENERAL U.S. MEDICAID POPULATION}

${ }^{1}$ Sarah K Chen, ${ }^{1}$ Medha Barbhaiya, ${ }^{3}$ Michael A Fischer, ${ }^{1}$ Hongshu Guan, ${ }^{1}$ Candace H Feldman, ${ }^{2}$ Brendan M Everett, ${ }^{1}$ Karen H Costenbader*. 'Divisions of Rheumatology, Immunology and Allergy, Department of Medicine, Brigham and Women's Hospital, Harvard Medical School, Boston, MA; ${ }^{2}$ Pharmacoepidemiology, Department of Medicine, Brigham and Women's Hospital, Harvard Medical School, Boston, MA; ${ }^{3}$ Cardiology, Department of Medicine, Brigham and Women's Hospital, Harvard Medical School, Boston, MA

\subsection{6/lupus-2018-Ism.54}

Background Both SLE and diabetes mellitus (DM) patients have elevated risks of atherosclerotic cardiovascular disease. Risk of heart failure (HF), an end-stage of cardiovascular disease and a leading cause of hospitalization in the U.S., is also elevated among DM patients, but has not been well studied in SLE. We investigated rates and risks of HF hospitalization among SLE patients compared to age- and sex-matched DM and general Medicaid patients.

Methods We used Medicaid Analytic eXtract (MAX) data, containing billing claims for Medicaid patients from the 29 most populated US states 2007-2010. We identified SLE and DM patients, ages 18-65, using $\geq 3$ ICD-9 codes for SLE or DM, each separated by $\geq 30$ days. Index date was $3 \mathrm{rd}$ diagnosis code. We matched each SLE patient at index date to $2 \mathrm{DM}$ patients and 4 general Medicaid patients without SLE or DM, by age at index date and sex. Baseline period was 6 months of continuous Medicaid enrollment prior to index date for all patients. Subjects were followed until death, disenrollment or end of follow-up. We used ICD-9 codes to identify HF hospital discharge diagnosis and calculated rates of first HF hospitalization event per 1000 person-years for each cohort. Cox proportional hazard models, accounting for competing risk of death, estimated hazard ratios (HR) for first HF hospitalization events. In a secondary analysis, we excluded those with baseline HF.

Results 40,212 SLE patients were matched to 80,424 DM and 160848 general patients. In all cohorts, 92\% were female, and mean age was $40.3( \pm 12.1)$ years. Mean follow-up was $1.8( \pm 1.1)$ years for SLE, $1.8( \pm 1.1)$ years for DM, and 1.6 $( \pm 1.2)$ years for general patients. Baseline CVD was present 